\title{
Synthesis and characterisation of PEG-peptide surfaces for proteolytic enzyme detection
}

\author{
Roza Trzcinska • Piotr Suder • Anna Bodzon-Kulakowska • \\ Magdalena Skalska • Andrzej Marcinkowski • Jerzy Kubacki • \\ Roman Pedrys $•$ Jerzy Silberring $•$ Andrzej Dworak $•$ Barbara Trzebicka
}

Received: 20 March 2013 / Revised: 10 May 2013 / Accepted: 17 May 2013 /Published online: 20 June 2013

(C) The Author(s) 2013. This article is published with open access at Springerlink.com

\begin{abstract}
Peptide surfaces were obtained by the covalent immobilisation of fluorescently labelled pentapeptides carboxyfluorescein-glycine-arginine-methionine-leucine-glycine, either directly or through a poly(ethylene glycol) (PEG) linker on modified silicon wafers. Each step during the preparation of the peptide surfaces was confirmed by several surface characterisation techniques. Time-of-flight secondary ion mass spectrometry (ToF-SIMS) and X-ray photoelectron spectroscopy were used to determine the surface composition, the wafers philicity was measured by contact angle and atomic force microscopy was used to investigate the surface morphology. Exposure of the peptide surfaces to trypsin resulted in the release of a fluorescently labelled peptide product, which allowed the kinetics of the enzymatic reaction to be followed with the aid of fluorescence spectroscopy. The electrospray
\end{abstract}

Published in the topical collection Separation and Characterization of Natural and Synthetic Macromolecules with guest editors Albena Lederer and Peter J. Schoenmakers.

Electronic supplementary material The online version of this article (doi:10.1007/s00216-013-7082-z) contains supplementary material, which is available to authorized users.

R. Trzcinska $\cdot$ A. Marcinkowski $\cdot$ J. Silberring $\cdot$ A. Dworak •

B. Trzebicka $(\bowtie)$

Centre of Polymer and Carbon Materials,

Polish Academy of Sciences, M. Curie-Sklodowskiej 34,

Zabrze 41-819, Poland

e-mail: btrzebicka@cmpw-pan.edu.pl

P. Suder $\cdot$ A. Bodzon-Kulakowska $\cdot$ J. Silberring

AGH University of Science and Technology, Mickiewicza 30,

Krakow 30-059, Poland

M. Skalska $\cdot$ R. Pedrys

Faculty of Physics, Astronomy and Applied Computer Science,

Jagiellonian University, Reymonta 4,

Krakow 30-059, Poland

J. Kubacki

Institute of Physics, University of Silesia, Uniwersytecka 4,

Katowice 40-007, Poland ionisation mass spectrometry analysis of the post-digestion solution confirmed that the pentapeptides attached to the solid support undergo specific trypsin hydrolysis at the Cterminus of the arginine residues. Detailed surface analyses before and after the enzyme action was performed using ToF-SIMS. Because of the limited accessibility of the short peptide directly attached to the surface, a quantitative yield of enzymatic hydrolysis was observed only in case when the peptide was bound through the PEG linker. The insertion of the PEG linker increased the number of immobilised peptides and the rate of enzymatic digestion which consequently improved the quality of the enzyme assays. The described approach may be used for different peptide sequences designed for other proteases.

Keywords Interface/surface analysis $\cdot$ Polymers $\cdot$ Mass spectrometry/ICP-MS · Amino acids/peptides $\cdot$ Biomaterials

\section{Introduction}

Recently, peptide surfaces have received considerable attention and have found many applications in the biological and pharmaceutical fields, e.g. in protein detection [1], enzyme assays [2], protein interactions studies [3], rapid screening for selective substrates and inhibitors [4], and drug profiling [5]. The main advantage of using a peptide array system is the possibility for simultaneously screening thousands of binding events in biological and pharmaceutical research [6]. Such applications require that peptide surfaces ensure the optimal binding capacity of the peptide substrate, retain its biological activity and provide the accessibility of the substrate to enzyme action. The surface should also minimise non-specific binding of biomolecules.

The above requirements can be achieved by selecting proper surface parameters and detection techniques. The surface homogeneity, roughness, philicity, density of functional 
groups and the spacing between surface and biologically active compounds may be crucial for study of enzymes with aid of peptide surfaces. Current detection strategies can be classified into label-free methods, such as mass spectrometry [7], surface plasmon resonance [8], atomic force microscopy [9] and labelled probe methods, such as fluorescence [10], chemiluminescence [11] and radioactivity detection [12]. The correct combination of the applied analytical methods is essential for enzyme recognition.

Considerable efforts have been devoted to preparing various types of supports and to developing an optimal method for attaching peptides to the surface [13]. The rigid materials used as supports have many advantages over porous ones-ensuring immediate access of enzyme to the peptide, improving image acquisition and allowing for the use of mass spectrometry as a detection technique [14]. The major methods for immobilising bioactive compounds on a surface are as follows: adsorption [15], ligand-receptor pairing [16] and covalent immobilisation [17] on the chip. Adsorption methods, despite their simplicity, have some drawbacks; the main is a risk for the spontaneous removal of adsorbed peptides during experimental steps. The covalent attachment of bioactive compounds to the functionalised surface results in the formation of the most stable bonds.

The peptides can be attached to the support directly or by using a spacer. It has been reported that the presence of poly(ethylene glycol) (PEG) as a linker between the surface and the peptide improved the assays [18-20]. The presence of hydrophilic PEG chains reduces the non-specific binding of biomolecules to the surface, provides a low background during fluorescence measurements, minimises steric hindrance and consequently makes the linked peptide fully accessible for enzyme actions.

Proteases are a large group of enzymes that are able to catalyse the hydrolysis of appropriate peptide bonds [21]. These enzymes regulate protein functions in the body and are involved in a multitude of physiological processes, from simple digestion of food proteins to highly regulated cascades [22]. Therefore, aberrations in the expressions or functions of proteases have been implicated in many pathological conditions, which make many proteolytic enzymes of clinically relevant importance valuable as diagnostic biomarkers. Peptide surfaces in combination with some detection techniques enabled the development of tools for identification of enzyme in human serum [23].

In this work, we proposed a simple method for preparing a well-characterised peptide-containing surface as an effective tool for studying the action of trypsin and showed the proper characterisation way for determining both the surface structure and composition. As a trypsin substrate, the pentapeptide sequence carboxyfluorescein-glycine-arginine-methionine-leucine-glycine (CF-GRMLG) was used. Labelling of peptide by carboxyfluorescein $(\mathrm{CF})$ allowed for fluorescence detection of the enzymatic reaction products. The peptides were covalently bound to the modified silicon surface directly or through a PEG linker. Time-of-flight secondary ion mass spectrometry (ToF-SIMS), X-ray photoelectron spectroscopy (XPS), contact angle (CA) and atomic force microscopy (AFM) measurements were used to confirm each step during the preparation of the peptide surfaces. The influence of short PEG linker on the quality of performed enzyme assay was shown. The products from the trypsin hydrolysis of the peptide surfaces were studied in detail using electrospray ionisation mass spectrometry (ESIMS), fluorescence and ToF-SIMS to confirm the usefulness of the prepared wafers as an analytical platform to study proteases.

\section{Materials and methods}

\section{Materials}

Boron-doped, p-type silicon wafers (100) with a thickness of $525 \mu \mathrm{m}$ were supplied by CEMAT SILICON S.A. (Poland). 1-(9-Fluorenylmethyloxycarbonyl)amino-3,6,9,12,15,18, 21,24,27,30,33,36-dodecaoxanonatriacontan-39-oic acid (Fmoc-NH-PEG(6)-COOH, 98 \%) and Fmoc-G-2-chlorotrityl polystyrene resin were purchased from Iris Biotech $\mathrm{GmbH}$ (Germany). 2-Chlorotrityl chloride resin was purchased from Rapp Polymere (Germany). Hydroxybenzotriazole hydrate (HOBt, $98 \%$ ) was purchased from Advanced ChemTech (USA). Water (gradient grade) and acetonitrile (gradient grade, $99.9 \%$ ), sulphuric acid (96\%), hydrogen peroxide (30\%), ethanol (99.8\%), methanol $(\mathrm{MeOH}, 99.8 \%)$, dichloromethane (DCM, $99.8 \%$ ), hexane (99 \%), N,Ndimethylacrylamide (DMF, $99.8 \%$ ), diethyl ether (pure p.a.), $n$-buthanol (pure p.a.), phenol (pure p.a.) and potassium hydroxide were obtained from POCh (Poland). Trypsin (sequencing grade) was purchased from Promega (USA). Fmoc L-amino acids (Fmoc-G, Fmoc-L, Fmoc-M, Fmoc$\mathrm{R}(\mathrm{Pbf}) ; \geq 98 \%$ ), (5,6)-carboxyfluorescein ( $\mathrm{CF}, \geq 95 \%$ ), acetic acid (AcOH, $99 \%$ ), trifluoroethanol (TFE, $99 \%$ ), trifluoroacetic acid (TFA, $99 \%$ ), triisopropylsilane (TIS, $99 \%$ piperidine (99\%), $N, N$-diisopropylcarbodiimide, (1cyano-2-ethoxy-2-oxoethylidenaminooxy)dimethylaminomorpholino-carbenium hexafluorophosphate (COMU, $97 \%$ ), $N, N$-diisopropylethylamine (DIPEA, $99 \%$ ), diisopropylcarbodiimide (DIC, $99 \%$ ), ammonium bicarbonate ( $\geq 99.5 \%$ ), 3-aminopropyltriethoxysilane (APTES, $99 \%$ ), potassium cyanide (BioUltra, $\geq 98.0 \%$ ) and ninhydrin ( $\geq 98.0 \%$ ) were purchased from Sigma-Aldrich. DMF was distilled under reduced pressure before use. DCM and acetone were distilled before use. Pyridine was distilled over potassium hydroxide. For the mass spectrometry measurements, MilliQ quality water was used. The other reagents were used as received. 
Synthesis of fluorescent peptide CF-GR(Pbf)MLG

Solid-phase peptide synthesis (SPPS), based on the Fmoc strategy [24], was performed to obtain the fully protected peptide. Fmoc amino acids were manually assembled on the Fmoc-G-2-chlorotrityl resin (380 mg, $0.59 \mathrm{mmol} / \mathrm{g})$. Stepwise coupling reactions were performed in DMF solution $(2 \mathrm{~mL})$ for $2 \mathrm{~h}$ using three equivalents of each amino acid (Fmoc-L (238 mg, 0.674 mmol), Fmoc-M (250 mg, 0.674 mmol), Fmoc-R(Pbf) (437 mg, 0.674 mmol), Fmoc-G (253 mg, $0.674 \mathrm{mmol})$ ), COMU (288 mg, $0.674 \mathrm{mmol}$ ) and six equivalents of DIPEA $(235 \mu \mathrm{L}, 1.348 \mathrm{mmol})$. The removals of the Fmoc were conducted using $5 \mathrm{~mL}$ of a $20 \%$ piperidine solution in DMF (two times for $10 \mathrm{~min}$ ). After each coupling and deprotection step, the resin was washed three times with DMF, DCM and then again with DMF. To introduce $\mathrm{CF}$ to the N-terminal amine group of the last glycine residue, three equivalents of CF (253 mg, 0.674 $\mathrm{mmol})$, HOBt (103 mg, $0.674 \mathrm{mmol})$ and DIC $(110 \mu \mathrm{L}$, $0.674 \mathrm{mmol}$ ) in $2 \mathrm{~mL}$ DMF were added to the resin suspension. After $2 \mathrm{~h}$ of reaction, the resin was additionally washed (two times for $10 \mathrm{~min}$ ) with $20 \%$ piperidine in DMF to remove the phenyl esters formed by the acylation of the CF phenolic hydroxyls. The product was cleaved from the solid support using a 30-min treatment of the peptide-loaded resin in a $5-\mathrm{mL}$ mixture composed of TFE/AcOH/DCM (1:1:8, $v / v / v)$. Next, the resin was filtered and washed with the cleavage solution (two times for $5 \mathrm{~min}$ ). One hundred and fifty millilitres of hexane was added to the mixture to remove acetic acid as an azeotrope with hexane. The solvents were removed with a rotary evaporator under reduced pressure. One hundred and fifty milligrams of the peptide CFGR(Pbf)MLG was obtained. The peptide was used without further purification.

\section{Modification of silicon surface}

The silicon wafers (100) were modified by silinisation to introduce the reactive amine groups onto the surface using a method similar to that described in literature (see ESM) [25]. Briefly, silicon wafers were treated with piranha solution, then with $2 \%$ APTES in ethanol. The obtained surface is described as Si-APTES.

To introduce PEG monolayer, the Si-APTES surfaces were immersed in a $0.25 \mathrm{M}$ DMF solution of 1-(9fluorenylmethyloxycarbonyl)amino-3,6,9,12,15,18,21,24, 27,30,33,36-dodecaoxanonatriacontan-39-oic acid (Fmoc-NHPEG-COOH, DP=6) with COMU $(0.25 \mathrm{M})$ and DIPEA $(0.5 \mathrm{M})$. The reaction was performed for $4 \mathrm{~h}$. After the reaction, the wafers were rinsed three times with DMF, three times with $\mathrm{MeOH}$ and dried under an argon flow. The Fmoc groups were removed by a $30-$ min treatment with $30 \%$ piperidine in DMF. Next, the wafers were rinsed three times with DMF, $\mathrm{MeOH}$ and dried under an argon flow. The obtained surface is described as Si-PEG.

Immobilisation of peptide

To immobilise the peptides on Si-APTES and Si-PEG surfaces, the wafers were immersed for $16 \mathrm{~h}$ in a $0.2 \mathrm{M}$ peptide DMF solution in presence of COMU $(0.2 \mathrm{M})$ and DIPEA $(0.4 \mathrm{M})$. After the reaction, the peptide-bound surfaces were washed three times with $\mathrm{DMF}, \mathrm{MeOH}$ and dried under an argon flow. Next, the wafers were treated for $4 \mathrm{~h}$ with a mixture of TFA/TIS/phenol/water $(88 / 5 / 2 / 5, v / v / v / v)$ to remove the protecting Pbf groups of the arginine residues. After deprotection, the peptide surfaces were rinsed three times with DMF, DCM, MeOH and then dried under an argon flow. Surfaces were further described as $\mathrm{Si}$ GLMR(Pbf)G-CF and Si-PEG-GLMR(Pbf)G-CF.

\section{Trypsin hydrolysis}

Fifty microliters of a $50 \mathrm{mM}$ ammonium bicarbonate buffer containing $0.1 \mathrm{pmol}$ of trypsin was spotted onto each wafer and left overnight. The post-digestion solutions were collected and analysed using ESI-MS and fluorescence spectroscopy. Next, the surfaces were thoroughly washed with $\mathrm{MeOH}$ and dried under an argon flow.

\section{Fluorescence spectroscopy}

Fluorescence spectra were recorded on a Hitachi F-2500 fluorescence spectrophotometer at room temperature with excitation at $494 \mathrm{~nm}$ and emission at $520 \mathrm{~nm}$, characteristic for the carboxyfluorescein. The excitation and emission bandwidths were both $5 \mathrm{~nm}$. The kinetics of the trypsin hydrolysis was followed in situ in the spectrophotometer. One millilitre of a $50 \mathrm{mM}$ ammonium bicarbonate buffer containing $20 \mathrm{pmol}$ of trypsin was added to the cuvette that contained peptide surface. The fluorescence emission of the solution was measured for $5 \mathrm{~h}$ of reaction and recorded every $10 \mathrm{~s}$.

UV-Vis spectroscopy

A Jasco V-530 UV-Vis spectrophotometer was used.

Atomic force microscopy

The surface morphology studies were performed using a Multi-Mode AFM microscope with a NanoScope 3D controller (di-Veeco Instruments Inc, USA, CA), which was operated in tapping mode in air with standard $125 \mathrm{~mm}$ single-crystal silicon cantilevers (Model TESP, Veeco Instruments Inc., USA). Images of different scan sizes from $500 \mathrm{~nm}$ to $10 \mu \mathrm{m}$ were obtained using the piezoelectric 
scanner. Micrographs were recorded using the NanoScope Software V531r1, and the most representative images for each sample were selected from three measurements at different surface points. Root mean square of roughness value for each sample was averaged from three measurements in different cross-sections of the images.

\section{Contact angle}

The surface philicity measurements were performed using a CAM101 contact angle goniometer with an accuracy of $\pm 1^{\circ}$. The water contact angles were determined in air using the sessile-drop method. A 4- $\mu \mathrm{L}$ water droplet was placed on the surface and recorded for $30 \mathrm{~s}$. The contact angle value was taken as the average of five measurements at different parts of two surfaces prepared under the same experimental conditions.

\section{Electrospray ionisation mass spectrometry}

Mass spectra of the obtained peptides and the digestion products were collected on an AmaZon ETD (Bruker Daltonics, Bremen, Germany) mass spectrometer equipped with an ESI ion source. The mass spectrometer settings were as follows: mass spectra registered in a positive ion mode, capillary voltage of $-4,500 \mathrm{~V}$ and the temperature of the heated capillary was $280{ }^{\circ} \mathrm{C}$. The scans were registered in the MS enhanced resolution mode. The flow rate of the injected samples was set to $3 \mu \mathrm{L} / \mathrm{min}$. The solvent used for the experiments consisted of $30 \%$ methanol, $69.9 \%$ water and $0.1 \%$ formic acid $(v / v / v)$. The acquired spectra were analysed using Bruker Data Analysis software (ver. 3.0).

Time-of-flight secondary ion mass spectrometry

The experiments were performed using a ToF-SIMS V (ION-TOF GmbH, Münster, Germany) reflectron-type time-of-flight mass spectrometer equipped with a bismuth liquid metal ion gun. Pulsed $30 \mathrm{keV} \mathrm{Bi}_{3}{ }^{+}$clusters at an ion current of $\sim 0.61 \mathrm{pA}$ were used as primary ions. Positive secondary ions $(\mathrm{m} / \mathrm{z}, 1-860)$ were collected for individual $300 \mu \mathrm{m} \times 300 \mu \mathrm{m}$ areas. The mass spectra were internally calibrated using the $\mathrm{H}^{+}, \mathrm{H}_{2}{ }^{+}, \mathrm{CH}_{3}{ }^{+}, \mathrm{C}_{2} \mathrm{H}_{3}{ }^{+}, \mathrm{C}_{3} \mathrm{H}_{3}{ }^{+}$and $\mathrm{C}_{3} \mathrm{H}_{5}{ }^{+}$peaks. The $\mathrm{Bi}_{3}{ }^{+}$primary ion beam was randomly rastered in a pixel region of 128 by 128 pixels. A lowenergy electron flood gun was activated to neutralise the surface during the analysis. During static analysis, each point on the sample surface has a negligible probability of being hit by more than one primary ion. The total ion doses were maintained well below the static limit of $1 \times 10^{13}$ primary ions $/ \mathrm{cm}^{2}$. The same dose of $1 \times 10^{12}$ ions $/ \mathrm{cm}^{2}$ was applied for all of the surface analyses.
X-ray photoelectron spectroscopy

The XPS measurements were performed on a PHI5700/660 spectrometer using an $\mathrm{Al}-\mathrm{K} \alpha$ monochromatic $\mathrm{X}$-ray source with an energy of $1,486.6 \mathrm{eV}$ and an energy resolution of $0.3 \mathrm{eV}$. All photoelectron spectra were calibrated against the peaks of $\mathrm{Au} 4 \mathrm{f} 7 / 2$ at $83.98 \mathrm{eV}, \mathrm{Ag} 3 \mathrm{~d} 5 / 2$ at $368.27 \mathrm{eV}$ and $\mathrm{Cu}$ $2 \mathrm{p} 3 / 2$ at $932.67 \mathrm{eV}$ of binding energy. The quantitative surface composition (atom\%) was calculated for each element using the areas of the peaks.

\section{Results and discussion}

The synthetic procedure leading to well-defined surfaces with peptide or PEG-peptide monolayers responsive to trypsin hydrolysis is described below. The proper assortment of surface analytical techniques allowed confirming the chemical structure of the silicon surface after modification steps and the trypsin digestion of the peptides.

\section{Synthesis of fluorescent peptide CF-GR(Pbf)MLG}

The synthetic route leading to CF-GR(Pbf)MLG pentapeptide is schematically shown in the Fig. 1a (details are given in the experimental section).

Solid-phase peptide synthesis was conducted according to the active ester method using COMU in the presence of DIPEA [26]. All coupling steps were confirmed by the negative result of the Kaiser test [27], thus eliminating the need for recoupling. Applied method for introduction of $\mathrm{CF}$

a

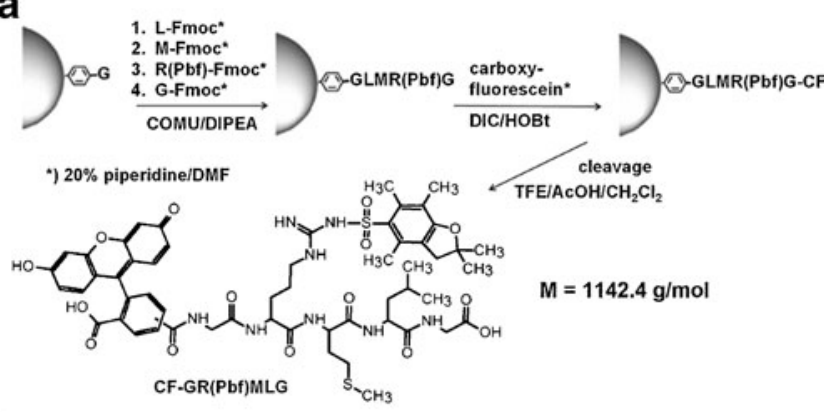

b

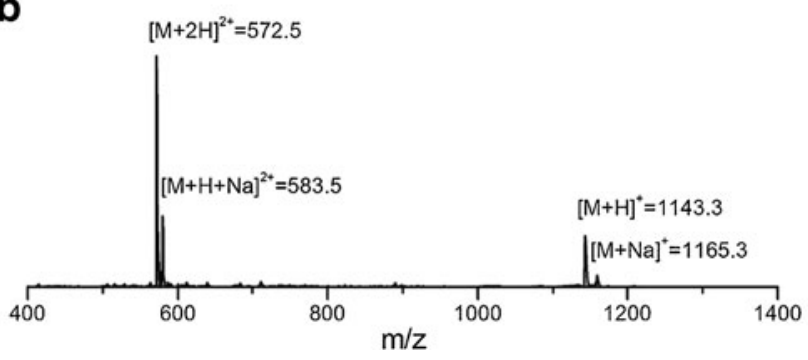

Fig. 1 a The scheme of pentapeptide CF-GR(Pbf)MLG synthesis. b The ESI-MS spectrum of CF-GR(Pbf)MLG pentapeptide 
led to the fluorescent product of the defined chemical structure. The use of highly acid-labile 2-chlorotrityl chloride polystyrene resin as a support for peptide synthesis allowed for cleavage of the peptide under very mild acidic conditions providing protected form CF-GR(Pbf)MLG.

The structure of the CF-GR(Pbf)MLG pentapeptide ( $M=$ $1,142.4 \mathrm{~g} / \mathrm{mol}$ ) was analysed by ESI-MS (Fig. 1b).

Two abundant signals of singly and doubly protonated ions, $[M+\mathrm{H}]^{+}(m / z=1,143.4)$ and $[M+2 \mathrm{H}]^{2+}(m / z=572.5)$, are present in the spectrum. The less intense signals at $\mathrm{m} / \mathrm{z}=$ $1,165.3$ and 583.5 result from the sodium adducts of the pseudo-molecular ions $[M+\mathrm{Na}]^{+}$and $[M+\mathrm{HNa}]^{2+}$. The purity of the obtained pentapeptide exceeded $97 \%$, as indicated by reverse-phase HPLC (see Electronic Supplementary Material, Fig. S1).

Both the ESI-MS and reverse-phase HPLC analyses confirmed the successful peptide synthesis protocol. The use of the coupling agent COMU, which was recently described by ElFaham [26], resulted in the formation of a well-defined peptide with high purity. Only one reactive carboxyl group contained in the peptide structure provides the possibility for site-specific binding of the peptide onto the amine-rich surfaces.

Modification of the silicon surface for peptide attachment

To enable peptide immobilisation, the silicon surface was modified in several consecutive steps to introduce the active amino groups.

The amino-silylation of silicon wafers using 3-amino propyltrietoxysilane led to Si-APTES surface (Fig. 2a, surface 1) with $3.5 \mathrm{pmol} / \mathrm{mm}^{2}$ amine groups (Electronic Supplementary Material). The applied synthetic procedure was similar to methods described in the other works [25]. Obtained density of amine groups are close to the values reported for a silane-modified silicon surface [4].

The prepared Si-APTES silicon wafers were used for the direct immobilisation of the CF-GR(Pbf)MLG pentapeptide or subjected to further modification with PEG.
Fig. 2 a Grafting of poly(ethylene glycol) to the $\mathrm{Si}$ APTES surface. b Fragments of the ToF-SIMS spectra for the Si-APTES, Si-PEG-Fmoc and Si-PEG surfaces
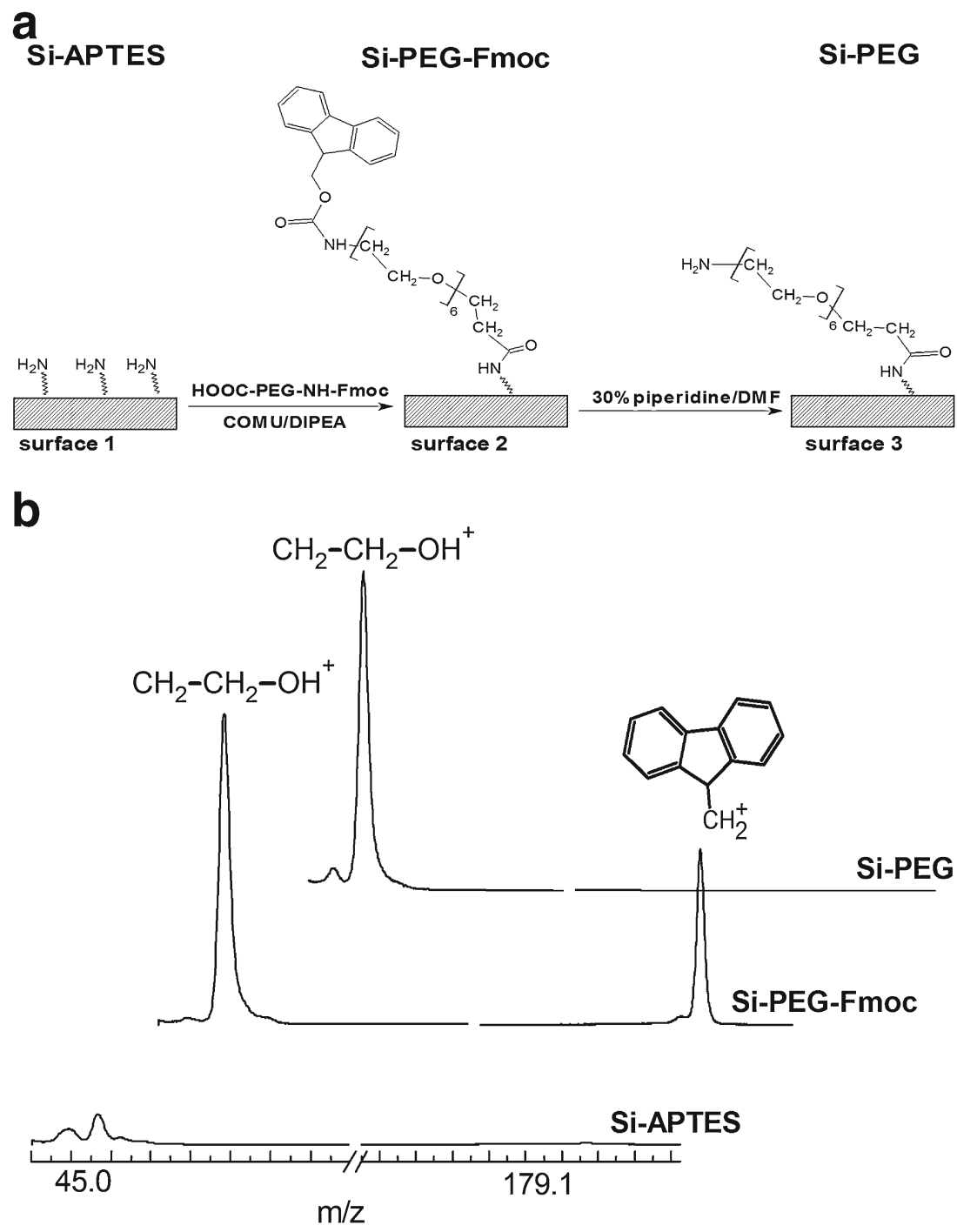
To obtain a PEG monolayer on the silicon surface, we applied a heterobifunctional, commercially available HOOCPEG(6)-NH-Fmoc which contains six ethylene glycol units $(\mathrm{DP}=6)$ and two end-groups: carboxyl group and protected by Fmoc amine group. The PEG end-functionalities were selected to provide chemoselective grafting to the support without side reactions, such as reactions between the PEG chains in solution or the formation of loops on the surface by bounding both chain ends.

The PEG monolayer was formed through the condensation of the carboxyl end-groups of HOOC-PEG(6)-NHFmoc and the amines present on the Si-APTES surface (Fig. 2a, surface 2, Si-PEG-Fmoc) followed by Fmoc removal (Fig. 2a, surface 3, Si-PEG).

ToF-SIMS analysis directly confirmed the introduction of the PEG monolayer onto the silicon wafers (Fig. 2b).

In the ToF-SIMS spectrum of Si-PEG-Fmoc, a characteristic signal which represents ethylene glycol repeating unit $\left[\mathrm{C}_{2} \mathrm{H}_{5} \mathrm{O}\right]^{+}$at $m / z=45.0$ ion was significantly increased in comparison with Si-APTES. Moreover, the signal $\left[\mathrm{C}_{14} \mathrm{H}_{11}\right]^{+}$ at $m / z=179.1$ from the Fmoc modification appeared in the spectrum, thus proving the attachment of Fmoc-terminated PEG. Similar fragments were observed by Ulijn and coworkers for ToF-SIMS analysis of PEG-containing glass surfaces [28]. The removal of the Fmoc-protecting group with piperidine leading to Si-PEG surface resulted in a complete disappearance of the $m / z=179.1$ ion, which indicated a complete deprotection of PEG, whereas the intensity of the signal at $m / z=45.0 \mathrm{did}$ not change, proving that the PEG chains remained attached to the surface.

The chemical modification of the silicon surface resulted in changes in the surface morphology and philicity (see Electronic Supplementary Material, Fig. S5). The surface roughness significantly increased after grafting the PEGFmoc monolayer to the surface. By comparing the contact angle values of the amino-terminated surfaces of Si-APTES and Si-PEG, it can be observed that the presence of the PEG linker between the surface and the amine groups increased the surface hydrophilicity.

The ToF-SIMS spectra, changes in the surface roughness and the contact angles confirmed the successful functionalisation of the silicon wafers.

Peptide surfaces and their characterisation

The peptide CF-GR(Pbf)MLG was immobilised directly on the Si-APTES surface (Fig. 3a, surface 4, Si-GLMR(Pbf)G$\mathrm{CF})$ or through a short poly(ethylene glycol) linker $(\mathrm{DP}=6)$ (Fig. 3b, surface 6, Si-PEG-GLMR(Pbf)LG-CF) under the same experimental conditions for both surfaces. The immobilisation was performed by the condensation of their carboxyl groups and the amines present on the surface using a newly developed, highly active reagent, COMU [26]. TFA treatment of peptide surfaces led to removal the protecting group of the arginine residue, which resulted in the peptide moiety being susceptible to the action of trypsin (Fig. 3a, surface 5, Si-GLMRG-CF; Fig. 3b, surface 7, Si-PEGGLMRLG-CF).

The peptide immobilisation increased the surface roughness compared to that of the initial Si-APTES and Si-PEG surfaces. The contact angle values after the introduction of the peptide moieties slightly decreased. Due to the hydrophilic character of the PEG, immobilisation of the peptide through PEG resulted in the surface having a more hydrophilic character, than when the peptide was bound directly to Si-APTES.

Surface composition of modified silicon wafers was verified by X-ray photoelectron spectroscopy (Table 1). The penetration depth of XPS measurement was deeper than the layer thickness and for this reason even for the outer layer strong signals from silicon are observed.

The amino-silylation process increased the amount of surface carbon due to the presence of carbon in the propyl group of the (3-aminopropyl)triethoxysilane. The surface concentration of nitrogen also increased what was connected with introduction of the amine groups onto the wafers surface. The oxygen and silicon concentrations decreased slightly as the silicon wafer signals were attenuated by (3-aminopropyl)silane overlayer. The increase of carbon and nitrogen signals for SiGLMRG-CF and Si-PEG-GLMRG-CF confirmed the immobilisation of the peptide. The signal from silicon with every modification step was reduced that indicate the growth of layer thicknesses. The comparison between atomic composition calculated and measured by XPS values are added to Electronic Supplementary Material (Table S1).

ToF-SIMS analysis was applied to prove the attachment of peptides to the surface. This provides an opportunity to detect molecules that are covalently bound to a solid support. For all of the analysed surfaces containing the pentapeptides, SiGLMR(Pbf)G-CF，Si-GLMRG-CF，Si-PEG-GLMR(Pbf)G$\mathrm{CF}$ and Si-PEG-GLMRG-CF, characteristic "immonium ions" $\mathrm{H}_{2} \mathrm{~N}^{+}=\mathrm{HC}-\mathrm{R}$, where $\mathrm{R}$ is the side chain group of the specific amino acid, related to glycine, arginine, leucine and methionine were visible in ToF-SIMS spectra in the low $\mathrm{m} / \mathrm{z}$ range as it is typical for peptides [29]. Due to the presence of carboxyfluorescein in the analysed samples, characteristic carboxyfluorescein fragments at $m / z=213.1,286.1,287.1$, 332.1, 376.1 and 388.1 were observed (Fig. 4a).

The ions from the carboxyfluorescein modification are more convenient for SIMS analysis than "immonium ions" because they are relatively intensive and appear in the high $\mathrm{m} / \mathrm{z}$ range, where the possibility for occurrence of other ions that have the same $\mathrm{m} / \mathrm{z}$ is negligible.

ToF-SIMS distribution maps of representative ions coming from the PEG $(m / z=45.0)$, Pbf $(m / z=189.1)$ and CF $(m / z=213.1,286.1,332.1)$ are shown in Fig. $4 b$. No characteristic signals coming from PEG or peptide are visible from 
a

Si-APTES

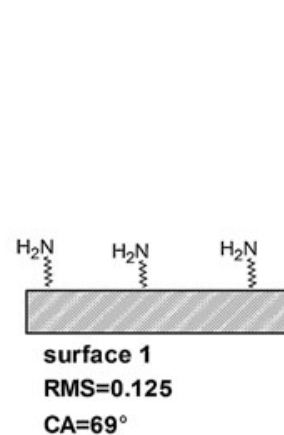

b

Si-PEG

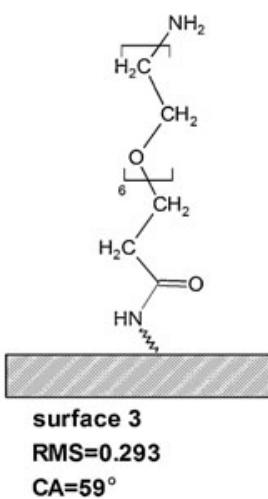

$\mathrm{Si}-\mathrm{GLMR}(\mathrm{Pbf}) \mathrm{G}-\mathrm{CF}$
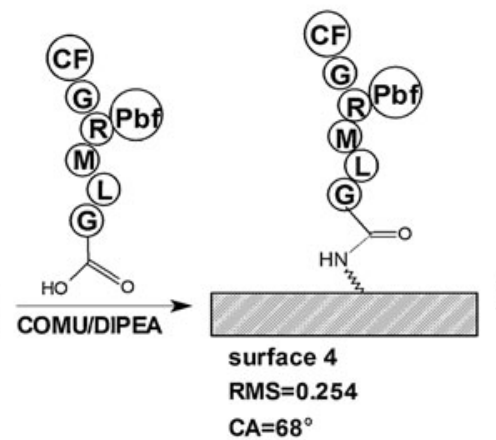

Si-PEG-GLMR(Pbf)G-CF

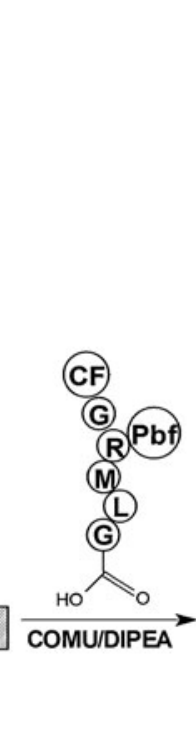

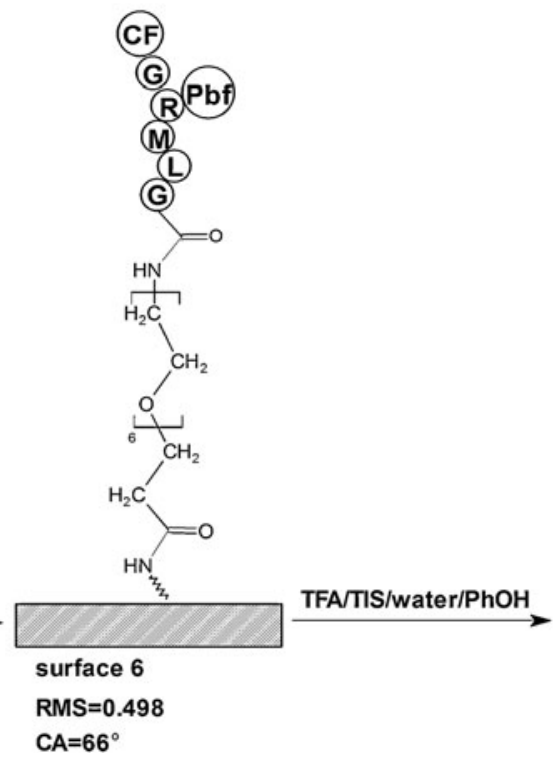

Si-GLMRG-CF

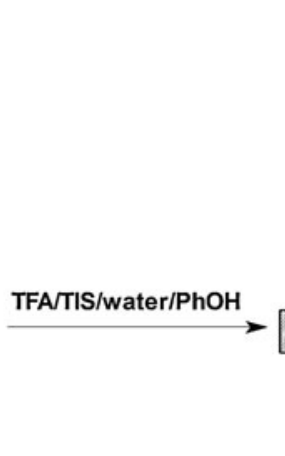

(CF)

G

(6)

(iM)

(L)

$\gamma=0$

$\mathrm{HN}_{3}$

surface 5
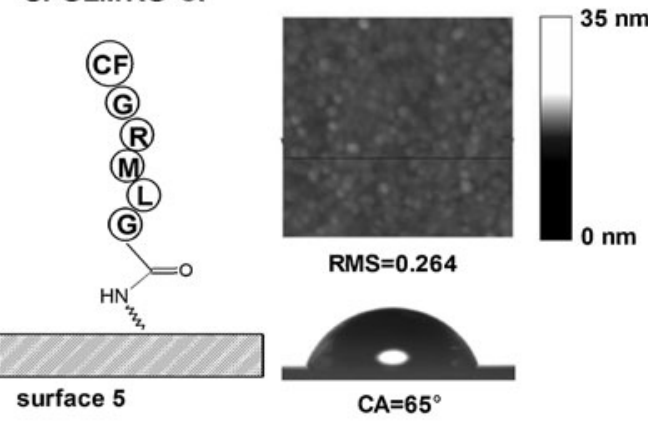

RMS $=0.264$

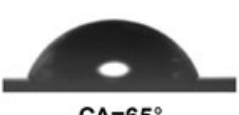

Si-PEG-GLMRG-CF

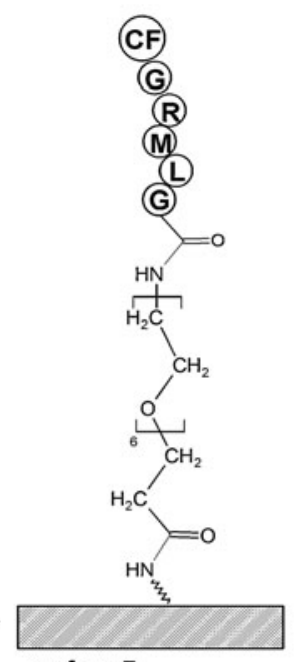

surface 7

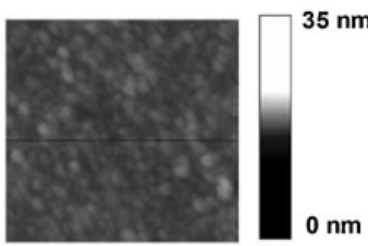

RMS $=0.420$

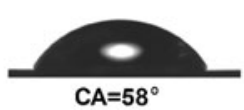

Fig. 3 Immobilisation of peptide a directly on the Si-APTES surface and $\mathbf{b}$ through a PEG linker on the Si-PEG surface. The AFM images and contact angles are shown

Si-APTES surface. Covering with PEG gives rise of the intensive signal $\left[\mathrm{C}_{2} \mathrm{H}_{5} \mathrm{O}\right]^{+}$at $m / z=45$ (rows $4,5,6$ ). The signal at $m / z=189.1$ assigned to the $\left[\mathrm{C}_{13} \mathrm{H}_{17} \mathrm{O}\right]^{+}$ion, which is indicative of $\mathrm{Pbf}$ protecting groups of arginine residues, appeared on surfaces Si-GLMR(Pbf)G-CF and Si-PEGGLMR(Pbf)G-CF covered with protected peptide (rows 2 and 5). After deprotection of the pentapeptides using trifluoroacetic acid, this ion completely disappeared (rows 3 and 6) indicating

Table 1 Surface atomic composition after certain modification step

\begin{tabular}{lcccc}
\hline Surface & $\begin{array}{l}\mathrm{C} \\
\text { [atom\%] }\end{array}$ & $\begin{array}{l}\mathrm{N} \\
\text { [atom\%] }\end{array}$ & $\begin{array}{l}\mathrm{O} \\
\text { [atom\%] }\end{array}$ & $\begin{array}{l}\mathrm{Si} \\
\text { [atom\%] }\end{array}$ \\
\hline Si-OH & 8.70 & 0.43 & 59.35 & 31.52 \\
Si-APTES & 21.94 & 2.31 & 48.96 & 26.79 \\
Si-GLMRG-CF & 26.20 & 2.95 & 46.04 & 24.80 \\
Si-PEG-GLMRG-CF & 28.71 & 3.71 & 44.09 & 23.49 \\
\hline
\end{tabular}

the quantitative removal of Pbf. The strong acidic conditions used for arginine deprotection did not influence the intensity of other ions (rows 3 and 6), ensuring that the prepared peptide surfaces are resistant to acid treatment. For all peptide containing surfaces (rows 2, 3, 5, 6), abundant signals at $m / z=213.1$, 286.1, and 332.1, which originate from carboxyfluorescein fragments, were present in the Tof-SIMS spectra. The intensities of the listed ions were higher in each case when the peptide was immobilised via a PEG spacer. All of the images indicate a homogeneous coating across the $300 \mu \mathrm{m} \times 300 \mu \mathrm{m}$ area.

The applied synthetic methods resulted in well-defined peptide surfaces. The described protocol for the immobilisation of peptides in their protected forms ensures the site-specific binding of peptide to the surface. The introduction of carboxyfluorescein dye to the pentapeptide moiety was helpful in the ToFSIMS analysis because under the primary ion beam $\mathrm{CF}$ released easily detectable, characteristic fragments. The applied method is universal and can be easily adapted to obtain various peptide 
a

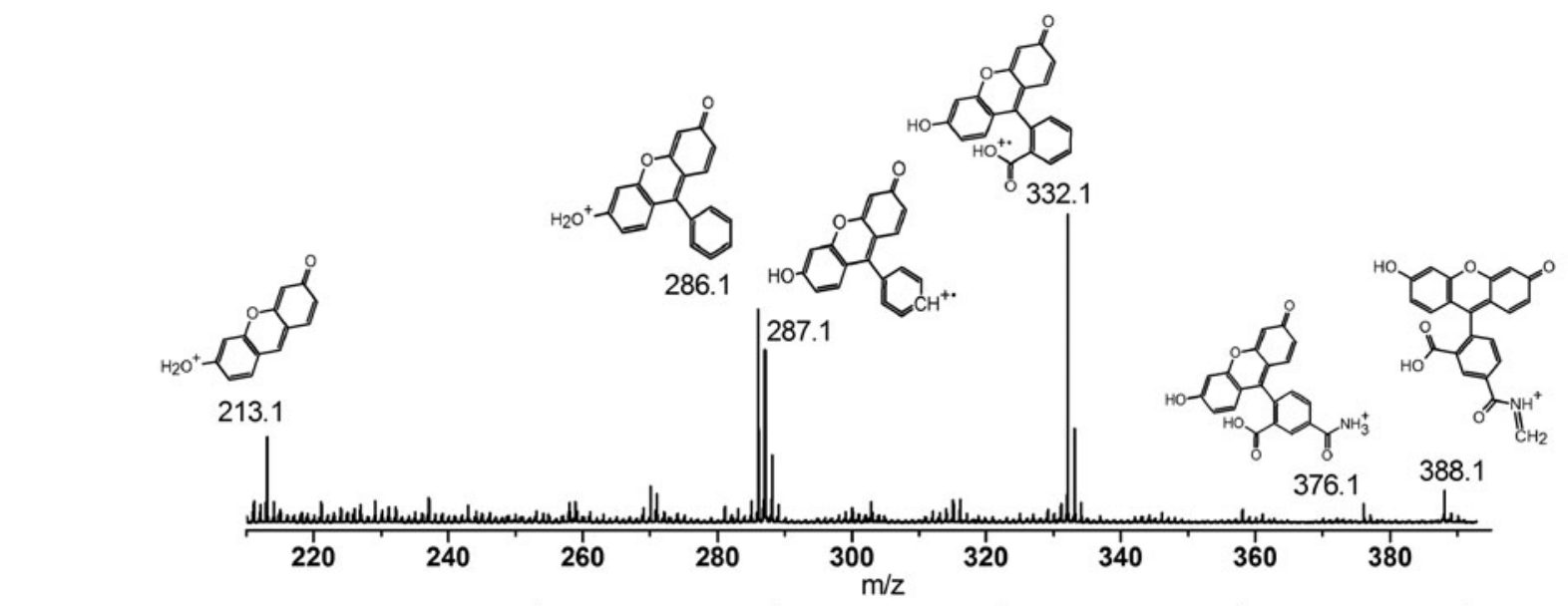

b
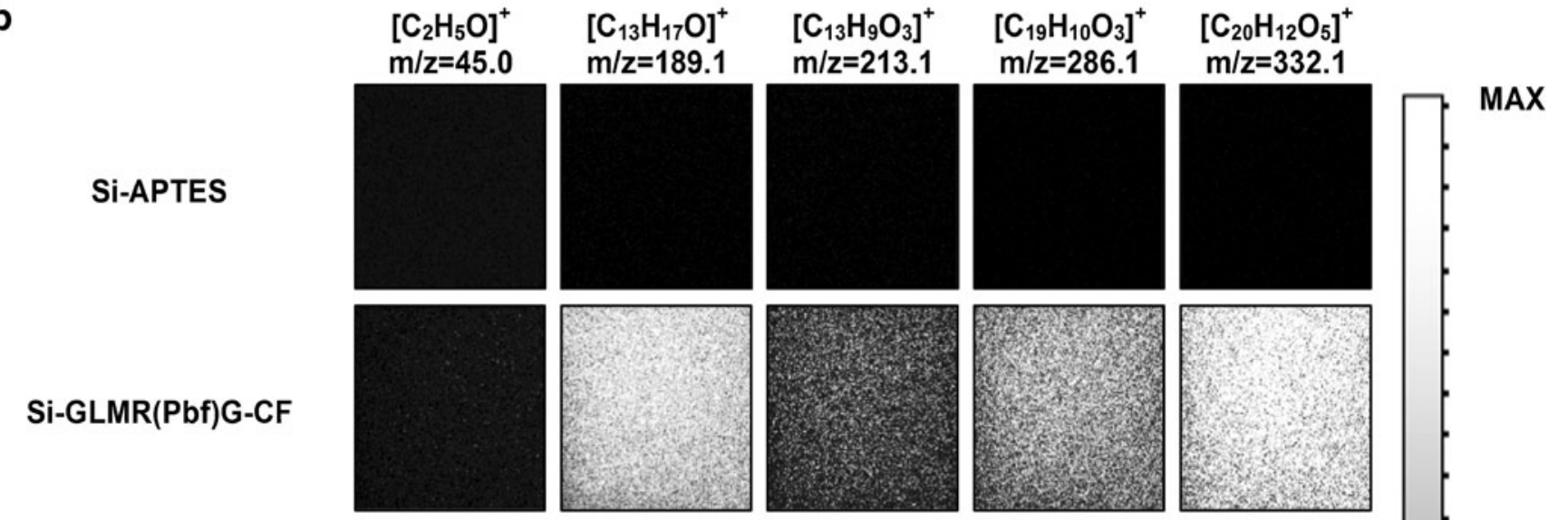

\section{Si-GLMRG-CF}
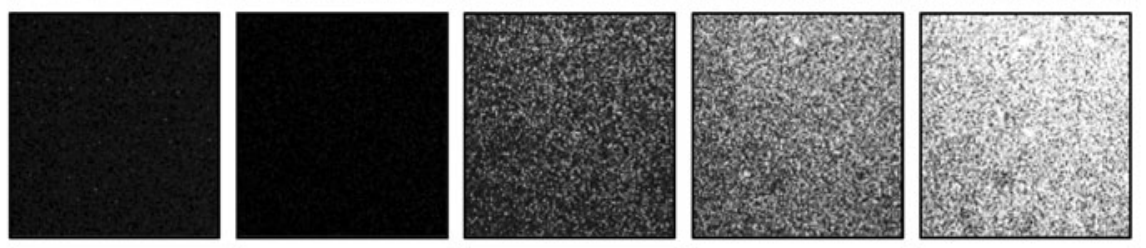

Si-PEG

\section{Si-PEG-GLMR(Pbf)G-CF}
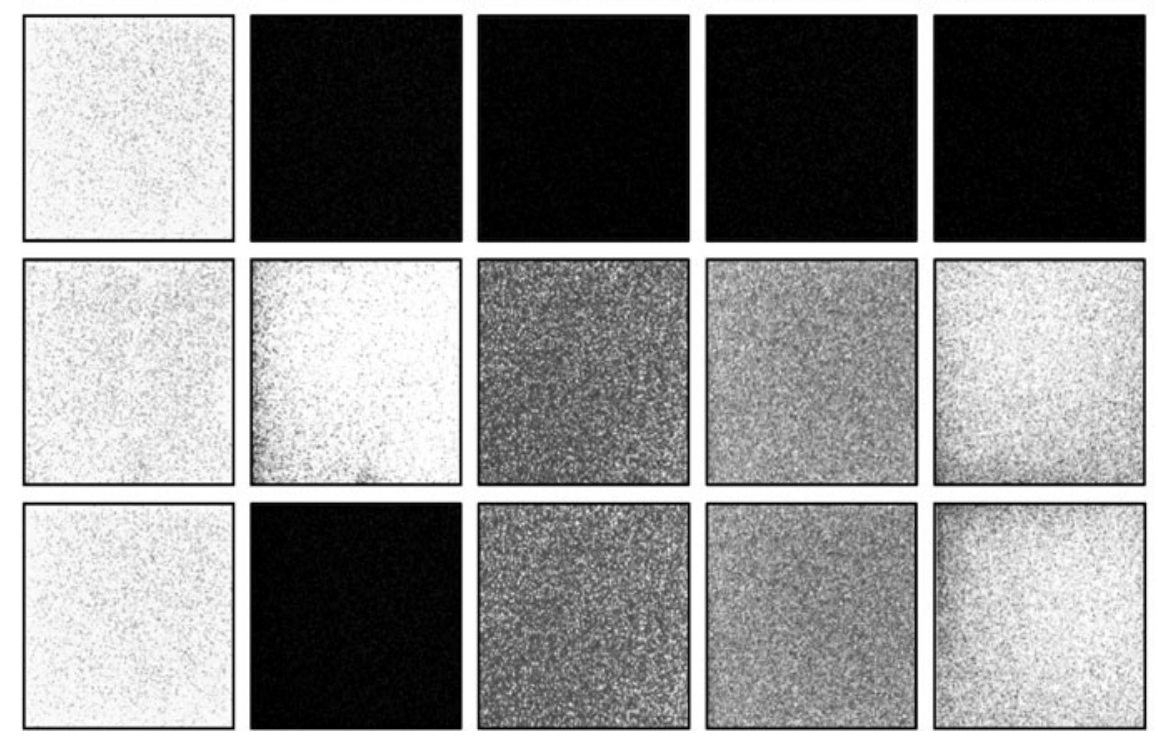

Fig. 4 a The fragment of the ToF-SIMS spectrum for the Si-PEG-GLMRG-CF surface. b Positive ion maps of chosen signals for all of the analysed surfaces $(300 \mu \mathrm{m} \times 300 \mu \mathrm{m})$; the intensity scale is the same for all ion images 
sequences on the modified silicon surfaces, including of multiple substrates (e.g. combinatorial chemistry). The obtained surfaces are stable. The wafers kept for 6 months in dark under nitrogen atmosphere show neither any changes in fluorescence nor ToF-SIMS measurements.

Mass spectrometry and fluorescence in following the trypsin action

Trypsin treatment of the Si-GLMRG-CF and Si-PEGGLMRG-CF surfaces resulted in the hydrolysis of peptide bond at $\mathrm{C}$-site of arginine and release of the CF-GR fluorescent peptide product, according to the scheme presented in Fig. 5a [30]. Study of the post-digestion solution (ESI-MS, fluorescence) and the surface (ToF-SIMS) can easily confirm the presence or absence of this enzyme in the sample.

The mass spectrometry analyses of the post-digestion solutions confirmed the specificity of the hydrolysis of the pentapeptide catalysed by trypsin (Fig. 5b).

The ESI-MS spectra of the solutions after incubation of peptide surfaces with trypsin exhibited an abundant signal at $m / z=295.6$. This ion is attributed to the doubly protonated pseudo-molecular ion $[M+2 \mathrm{H}]^{2+}$ of the expected digestion product CF-GR with a molar mass of $M=589.1 \mathrm{~g} / \mathrm{mol}$. The attraction of two protons was confirmed by its isotopic profile and was also confirmed by MS/MS studies (spectra not shown).

The on-chip tryptic hydrolysis of the CF-GRMLG pentapeptide on the Si-APTES and Si-PEG surfaces of the same area was monitored using fluorescence spectroscopy (Fig. 5c).

The emission values (excitation $\lambda=494 \mathrm{~nm}$ ) of the postdigestion solutions during trypsin hydrolysis revealed that the fluorescence intensity is considerably higher for the peptide immobilised through the PEG linker than for the peptide directly immobilised on the Si-APTES surface. To obtain the amount of released peptide, the CF-GR peptide was synthesised by SPPS and characterised by RP-HPLC (Electronic Supplementary Material, Fig. S2) and ESI-MS (Electronic Supplementary Material, Fig. S3). This peptide was used to create the fluorescence calibration curve (Electronic Supplementary Material, Fig. S4). It turned out that for the peptide attached directly, $0.14 \mathrm{pmol} / \mathrm{mm}^{2}$ CF-GR was released, while the peptide attached via PEG linker yielded $0.26 \mathrm{pmol} / \mathrm{mm}^{2}$, nearly a double amount (see ESM). The presence of PEG caused a faster rate of hydrolysis than that for the directly immobilised substrate. For the Si-PEGGLMRG-CF surface, the reaction was completed after $2 \mathrm{~h}$, whereas for the Si-GLMRG-CF surface, this process required more than $3 \mathrm{~h}$. Those results indicate that the peptides linked to the surface through PEG have higher affinity and accessibility to the enzyme than those directly immobilised on the Si-APTES layer. The applied analytical method using fluorescence for study enzyme action is very sensitive and allows for detection $0.01 \mathrm{pmol}$ released CF-GR product.
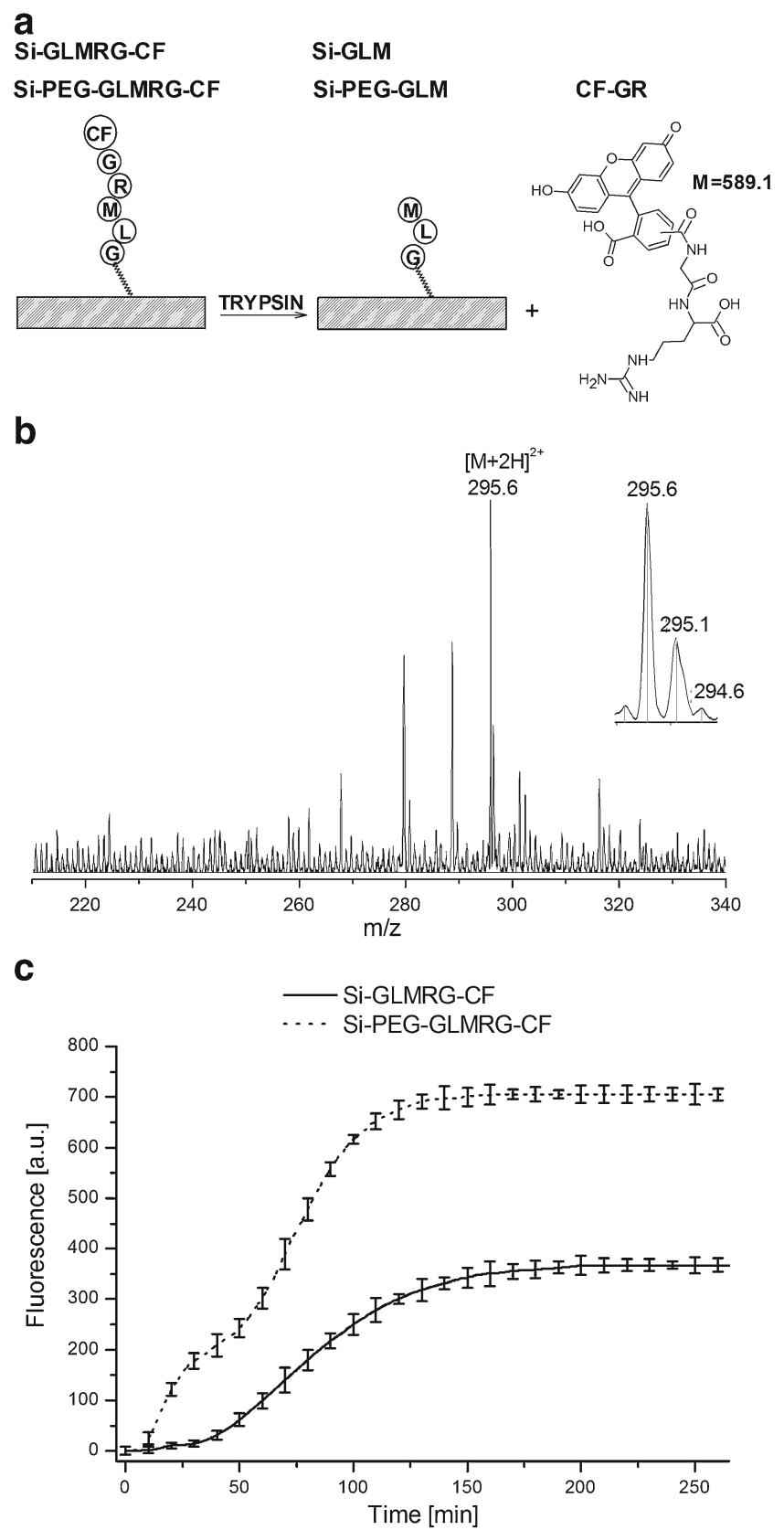

Fig. 5 a The scheme of the enzymatic hydrolysis of the peptide CFGRMLG attached to the Si-APTES or Si-PEG surface. b ESI-MS analysis of the post-digestion solution for Si-PEG-GLMRG-CF, where a signal at $m / z=259.6$ is a doubly protonated ion of a CF-GR product. $\mathbf{c}$ Fluorescence analysis of the time course of trypsin hydrolysis for $\mathrm{Si}$ GLMRG-CF and Si-PEG-GLMRG-CF surfaces $\left(\lambda_{\mathrm{em}}=520 \mathrm{~nm} ; \lambda_{\mathrm{ex}}=\right.$ $494 \mathrm{~nm})$. Results are means \pm SD for three independent experiments

The enzymatic hydrolysis was also studied by ToF-SIMS surface analysis. The maps for selected ions from the SiGLMRG-CF and Si-PEG-GLMRG-CF surfaces, both before and after exposure to trypsin, are shown in Fig. 6 a.

The representative ions for carboxyfluorescein $(\mathrm{m} / \mathrm{z}=$ 213.1, 286.1, 332.1) almost completely disappeared after enzymatic digestion what proved that exposure of the $\mathrm{Si}$ - 
Fig. 6 a Selected positive ion maps $(300 \mu \mathrm{m} \times 300 \mu \mathrm{m})$ from Si-GLMRG-CF and Si-PEGGLMRG-CF surfaces before and after trypsin digestion. $\mathbf{b}$ Comparison of the $m / z=332$ ion intensities before and after trypsin digestion of SiGLMRG-CF and Si-PEGGLMRG-CF surfaces a

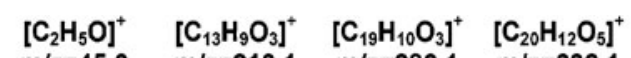

$\mathrm{m} / \mathrm{z}=45.0 \quad \mathrm{~m} / \mathrm{z}=213.1 \quad \mathrm{~m} / \mathrm{z}=286.1 \quad \mathrm{~m} / \mathrm{z}=332.1$

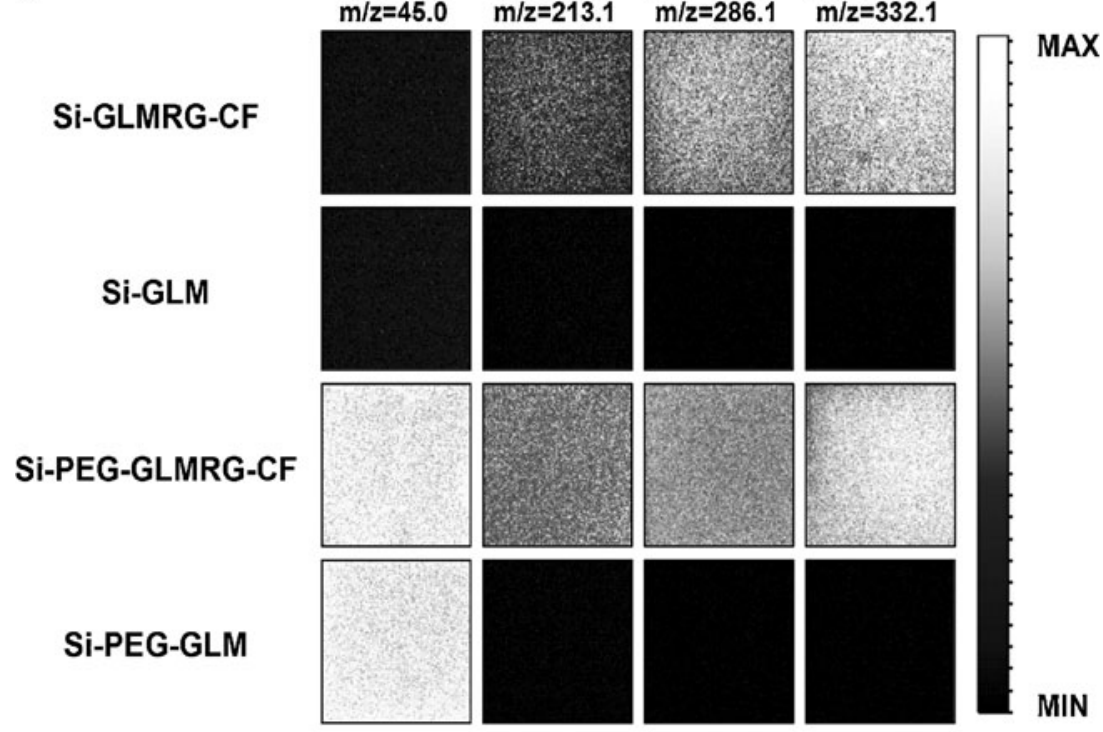

b

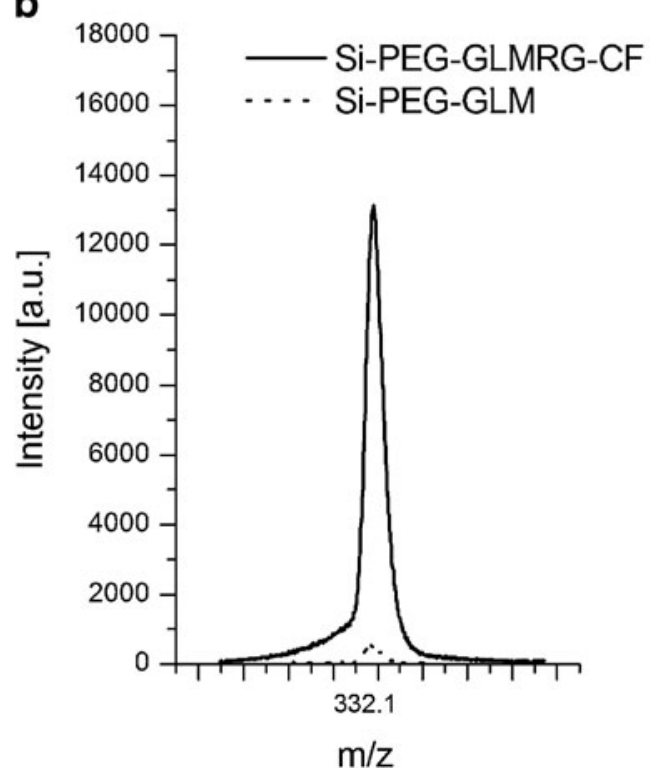

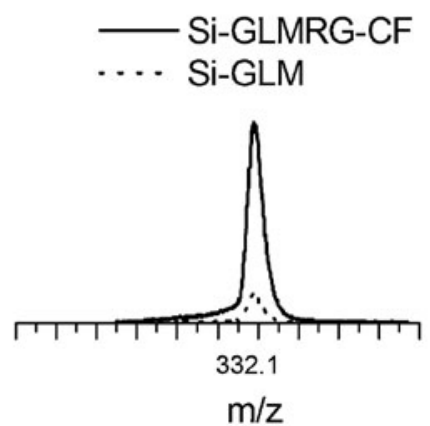

GLMRG-CF and Si-PEG-GLMRG-CF surfaces to trypsin resulted in efficient digestion process and removal of CF-GR product from the peptide surfaces. The ion at $m / z=45.0$ from the PEG-containing surface remained unchanged, which indicates that the hydrolysis process did not influence the PEG units.

To estimate the efficiency of trypsin hydrolysis, the ratios of the integrated representative signal at $m / z=332.1$ before and after digestion were compared (Fig. 6b). Although ToF-SIMS is not a quantitative method, the reduction in the abundance of the ion at $\mathrm{m} / \mathrm{z}=332.1$ clearly indicates the release of a CF-containing product as a result of tryptic digestion. The abundance of the ion was reduced by approximately $99 \%$ of the initial ion value for $\mathrm{Si}$ PEG-GLMRG-CF and by $85 \%$ for Si- GLMRG-CF. The exact amount of the released product cannot be determined, but the experiments revealed that immobilisation via PEG resulted in more efficient digestion process.
The significantly higher amount of released product in the case of Si-PEG-peptide surfaces cannot be exclusively caused by better accessibility (less steric hindrance) of peptide to enzyme. The ToF-SIMS indicates that the action of enzyme removes almost all CF-GR sequence fragments from the surface (99\% with PEG and $85 \%$ without PEG). The fluorescence measurements proved that much more CF-GR was digested from the surface containing linker, thus evidencing that the introduction of linker permits more peptides to be immobilised.

\section{Conclusions}

A short, fluorescently labelled peptide substrate CF-GRMLG was successfully used to prepare surfaces that are suitable for 
the efficient and easy detection of enzymes. The applied method for the preparation of peptide surfaces was effective and ensures site-specific peptide immobilisation directly onto the APTES-modified silicon surface or immobilisation through the PEG linker. The carboxyfluorescein residue connected to the peptide sequence appeared to be easily detectable not only for sensitive fluorescence analysis but also for ToF-SIMS identification, thereby providing several characteristic features for simplifying the reliable peptide recognition on the surface. Digestion of the peptide surfaces with trypsin led to the release of the fluorescent peptide fragment. The use of the obtained peptide surfaces enables enzymatic hydrolysis to be monitored on-chip, and its kinetics can be followed by fluorescence analysis. The ESI-MS measurement of the post-digestion solution confirmed the specificity of the enzymatic cleavage, whereas ToF-SIMS allowed for detailed surface analyses before and after the enzyme action. The presence of the PEG linker between the peptide and the surface facilitated the hydrolysis, making the applied peptides more prone to trypsin digestion than peptides directly attached to the surface. The peptide surfaces containing the PEG linker reach a higher peptide capacity, what increases sensitivity of trypsin assays. Furthermore, the immobilisation of the peptide through PEG led to an almost quantitate hydrolysis yield, whereas in the case of direct immobilisation, some undigested peptides were still present. The presence of short PEG spacer between the surface and peptide together with appropriate surface parameters, such as homogeneity, roughness, philicity and the density of functional groups resulted in the optimal analytical platform for the trypsin assay. Simple replacement of GLMRG by specific peptide sequences designed for other proteases (biomarkers) may provide a tool in the diagnosis of diseases and in monitoring the therapy. All the characterisation techniques used in work confirmed the usefulness of the prepared wafers as an analytical platform to study proteases.

Acknowledgments The authors acknowledge the Polish Ministry of Science and Higher Education Grant NN 209756740 for financial support. R. Trzcinska gratefully acknowledges the European Social Fund within the RFSD 2 project for financial support. The ToF-SIMS measurements has been performed at Institute of Physics, Jagiellonian University, Poland, with the equipment purchased thanks to the financial support of the European Regional Development Fund in the framework of the Polish Innovation Economy Operational Program (contract no. POIG.02.01.00-12-023/08).

Open Access This article is distributed under the terms of the Creative Commons Attribution License which permits any use, distribution, and reproduction in any medium, provided the original author(s) and the source are credited.

\section{References}

1. Reymond J-L, Wahler D (2002) Chem Bio Chem 3:701-708

2. Uttamchandani M, Moochhala S (2010) Biointerphases 5:FA24FA31

3. Katz C, Levy-Beladev L, Rotem-Bamberger S, Rito T, Rudiger SGD, Frirdler A (2011) Chem Soc Rev 40:2131-3145

4. Salisbury CM, Maly J, Ellman JA (2002) J Am Chem Soc 124:14868-14870

5. Reineke U, Schneider-Mergener J, Schutkowski M (2008) In: Ferrari M, Ozkan M, Heller MJ (eds) BioMEMS and biomedical nanotechnology. Springer, New York, 161-282

6. Reimer U, Reineke U, Schneider-Mergener J (2002) Curr Opin Biotechnol 13:315-320

7. Caputo E, Moharram R, Martin BM (2003) Anal Biochem 321:116-124

8. Johnsson B, Lofas S, Lindquist G (1991) Anal Biochem 198:268-277

9. Lee KB, Park SJ, Mirkin CA, Smith JC, Mrksich M (2002) Science 295:1702-1705

10. Zhu Q, Uttamchndani M, Li D, Lesaicherre L, Yao SO (1998) Org Lett 5:1257-1260

11. Schweitzer B, Predki P, Snyder M (2003) Proteomics 3:2190-2199

12. Falsey JR, Renil M, Park S, Li S, Lam KS (2001) Bioconjug Chem $12: 346-353$

13. Goddard JM, Hotchkiss JH (2007) Prog Polym Sci 32:698-725

14. Gurard-Levin ZA, Kim J, Mrksich M (2009) Chem Bio Chem 10:2159-2161

15. Haab BB, Dunham MJ, Brown PO (2000) Genome Biol 2:1-4

16. Zhu $\mathrm{H}$, Bilgin $\mathrm{M}$, Bangham R, Hall $\mathrm{D}$, Casamayor A, Bertone $\mathrm{P}$, Lan N, Jansen R, Bidlingmaier S, Houfek T, Mitchell T, Miller P, Dean RA, Gerstein M, Snyder M (2001) Science 293:2101-2105

17. Mac Beath G, Schreiber PL (2000) Science 289:1760-1763

18. Lesaicherre M, Uttamchandani M, Chen G, Yao S (2002) Bioorg Med Chem Lett 12:2079-2083

19. Janissen R, Oberbarnscheidt L, Oesterhelt F (2009) Coll Surf B: Bioint 71:200-207

20. Inamori $\mathrm{K}$, Kyo M, Matsukawa $\mathrm{K}$, Inoue $\mathrm{Y}$, Sonoda $\mathrm{T}$, Tatematsu K, Tanizawa K, Mori T, Katayama Y (2008) Anal Chem 80:643650

21. Barrett AJ, Rawlings ND, Woessner JF (eds) (2003) The handbook of proteolytic enzymes. Academic, London

22. Patel T, Gores GJ, Kaufmann SH (1996) FASEB J 10:587-597

23. Robinson WH, Steinman L, Utz PJ (2002) Biotechnics (Suppl) 33:66-69

24. Chan WC, White PD (eds) (2000) Fmoc solid phase peptide synthesis: a practical approach. Oxford University Press, New York

25. Cras JJ, Rowe-Taitt CA, Nivens DA, Ligler FS (1999) Bios Bioelectron 14:683-688

26. El-Faham A, Funosas RS, Prohens R, Albericio F (2009) Chem Eur J 15:9404-9416

27. Kaiser E, Colescott RL, Bossinger CD, Cook PI (1970) Anal Biochem 34:595-598

28. Todd SJ, Scurr DJ, Gough JE, Elexander MR, Ulijn RV (2009) Langmuir 25:7533-7539

29. Sole-Domenech S, Johansson B, Schalling M, Malm J, Sjovall P (2010) Anal Chem 82:1964-1974

30. Olsen JV, Ong S-E, Mann M (2004) Mol Cell Proteomics 3:608614 